\title{
A computational fluid dynamics simulation framework for ventricular catheter design optimization
}

\author{
Sofy H. Weisenberg, MSc, ${ }^{1}$ Stephanie C. TerMaath, PhD, ${ }^{1}$ Charlotte N. Barbier, PhD, ${ }^{2}$ \\ Judith C. Hill, PhD, ${ }^{3}$ and James A. Killeffer, MD ${ }^{4}$ \\ 'Department of Mechanical, Aerospace, and Biomedical Engineering, University of Tennessee, Knoxville; ${ }^{2}$ Instrument and \\ Source Division, Spallation Neutron Source, and ${ }^{3}$ Scientific Computing Group, National Center for Computational Sciences, \\ Oak Ridge National Laboratory, Oak Ridge, Tennessee; and ${ }^{4}$ Division of Neurosurgery, Department of Surgery, University of \\ Tennessee Graduate School of Medicine, Knoxville, Tennessee
}

OBJECTIVE Cerebrospinal fluid (CSF) shunts are the primary treatment for patients suffering from hydrocephalus. While proven effective in symptom relief, these shunt systems are plagued by high failure rates and often require repeated revision surgeries to replace malfunctioning components. One of the leading causes of CSF shunt failure is obstruction of the ventricular catheter by aggregations of cells, proteins, blood clots, or fronds of choroid plexus that occlude the catheter's small inlet holes or even the full internal catheter lumen. Such obstructions can disrupt CSF diversion out of the ventricular system or impede it entirely. Previous studies have suggested that altering the catheter's fluid dynamics may help to reduce the likelihood of complete ventricular catheter failure caused by obstruction. However, systematic correlation between a ventricular catheter's design parameters and its performance, specifically its likelihood to become occluded, still remains unknown. Therefore, an automated, open-source computational fluid dynamics (CFD) simulation framework was developed for use in the medical community to determine optimized ventricular catheter designs and to rapidly explore parameter influence for a given flow objective.

METHODS The computational framework was developed by coupling a 3D CFD solver and an iterative optimization algorithm and was implemented in a high-performance computing environment. The capabilities of the framework were demonstrated by computing an optimized ventricular catheter design that provides uniform flow rates through the catheter's inlet holes, a common design objective in the literature. The baseline computational model was validated using 3D nuclear imaging to provide flow velocities at the inlet holes and through the catheter.

RESULTS The optimized catheter design achieved through use of the automated simulation framework improved significantly on previous attempts to reach a uniform inlet flow rate distribution using the standard catheter hole configuration as a baseline. While the standard ventricular catheter design featuring uniform inlet hole diameters and hole spacing has a standard deviation of $14.27 \%$ for the inlet flow rates, the optimized design has a standard deviation of $0.30 \%$.

CONCLUSIONS This customizable framework, paired with high-performance computing, provides a rapid method of design testing to solve complex flow problems. While a relatively simplified ventricular catheter model was used to demonstrate the framework, the computational approach is applicable to any baseline catheter model, and it is easily adapted to optimize catheters for the unique needs of different patients as well as for other fluid-based medical devices. https://thejns.org/doi/abs/10.3171/2017.5.JNS161882

KEY WORDS ventricular catheter; design; shunt; optimization; computational fluid dynamics; hydrocephalus

$\mathrm{T}$ HE most common treatment for hydrocephalus patients is surgical insertion of a shunt that diverts cerebrospinal fluid (CSF) from the ventricular system to other areas of a patient's body where it is absorbed. While CSF shunts have proven effective at relieving the symptoms caused by elevated intracranial pressure, they have an alarmingly high rate of malfunction and failure, despite their relative simplicity compared with other medical devices. For example, over half of pediatric shunts require surgical intervention or revision, most within the 1st or 2nd year after the original shunt is implanted..$^{1,5,26}$ One major cause of shunt failure is obstruction of the ventricu-

ABBREVIATIONS CFD = computational fluid dynamics; CSF = cerebrospinal fluid; PEPT = positron emission particle tracking.

SUBMITTED July 19, 2016. ACCEPTED May 8, 2017.

INCLUDE WHEN CITING Published online November 10, 2017; DOI: 10.3171/2017.5.JNS161882. 
lar catheter. ${ }^{2,6,12,13,15,17,22,24}$ Studies investigating shunt survival rates have quantified that between $30 \%$ and $40 \%$ of pediatric shunt failures are caused by some form of shunt obstruction, the majority of which occur in the ventricular catheter. ${ }^{2,5,18,26}$ These obstructions are typically the result of migration and invasion of the choroid plexus into the inlet holes of the ventricular catheter, or less often, the result of aggregations of cells (glial cells, astrocytes, oligodendroglia, macrophages, and sometimes leukocytes), proteins (fibrin, albumin), pathological cells and tissues (inflammatory cells, red blood cells, platelets, and cell debris), blood clots, or combinations of these. . $^{213,15,19,23,27}$

Previous work ${ }^{3,7-11,15,20,27}$ suggests that the propensity for ventricular catheter obstruction is related to the fluid dynamics of the catheter and that CSF flow behavior into and through the catheter is highly dependent on catheter geometry. Such studies have demonstrated the need for a comprehensive correlation of the CSF flow profile to geometrical design variation. One approach to building this much-needed knowledge base is through parameter studies and sensitivity analysis, using validated 3D computational simulation to quantify the fluid dynamics for the evaluation of thousands of potential and interacting geometry modifications. A drawback to accomplishing such analyses, as noted by Galarza et al., ${ }^{8}$ is the time limitation in investigating the fluid dynamics for each new design and every possible geometrical variation. While computational simulation is an effective tool for evaluating designs prior to experimental and clinical testing, model development and computational mesh generation for each configuration is laborious and prohibits an all-encompassing evaluation of potential geometry modifications.

To overcome these time constraints, an open-source, computational framework for sensitivity analysis and design optimization has been developed, providing the capability for automated catheter model generation and calculation of the 3D flow profile via computational flu- id dynamics (CFD). In this framework, CFD is coupled with an optimization methodology, enabling rapid simulation of 3D CSF flow through ventricular catheters of varying geometries. A "black-box" coupling between 2 software packages allows for any validated 3D catheter model (unobstructed or obstructed) to serve as the baseline configuration, eliminating the time-consuming need to build a complex model for every geometrical variation on a given design. Implementing the framework on a supercomputer allows for the rapid, systematic investigation of a large number of geometry modifications for efficient design optimization and sensitivity analysis. Additionally, this framework is applicable to any parameterized baseline model and can be used to simulate complex fluid behavior, such as turbulent and pulsatile flow, fluid-structure interaction, and transient effects.

This framework is described and demonstrated on a common design objective from the literature: achieving uniform flow rates across the inlet holes of an unobstructed ventricular catheter with a typical quarter-symmetric hole configuration. A 3D flow model of this configuration was validated using 3D nuclear imaging and serves as the baseline for demonstrating the framework's capability to study the effects of geometry variation and design optimization relative to a desired flow objective.

\section{Methods}

\section{Computational Framework Overview}

The computational framework shown in Fig. 1 provides the capability to perform automated, iterative design exploration and optimization of a baseline catheter design. Two free, open-source software packages are coupled and implemented on a supercomputer to enable the rapid analysis of thousands of catheter design variations in a short amount of time. The Open Source Field Operation And Manipulation (OpenFOAM, Version 2.3.1, OpenCFD

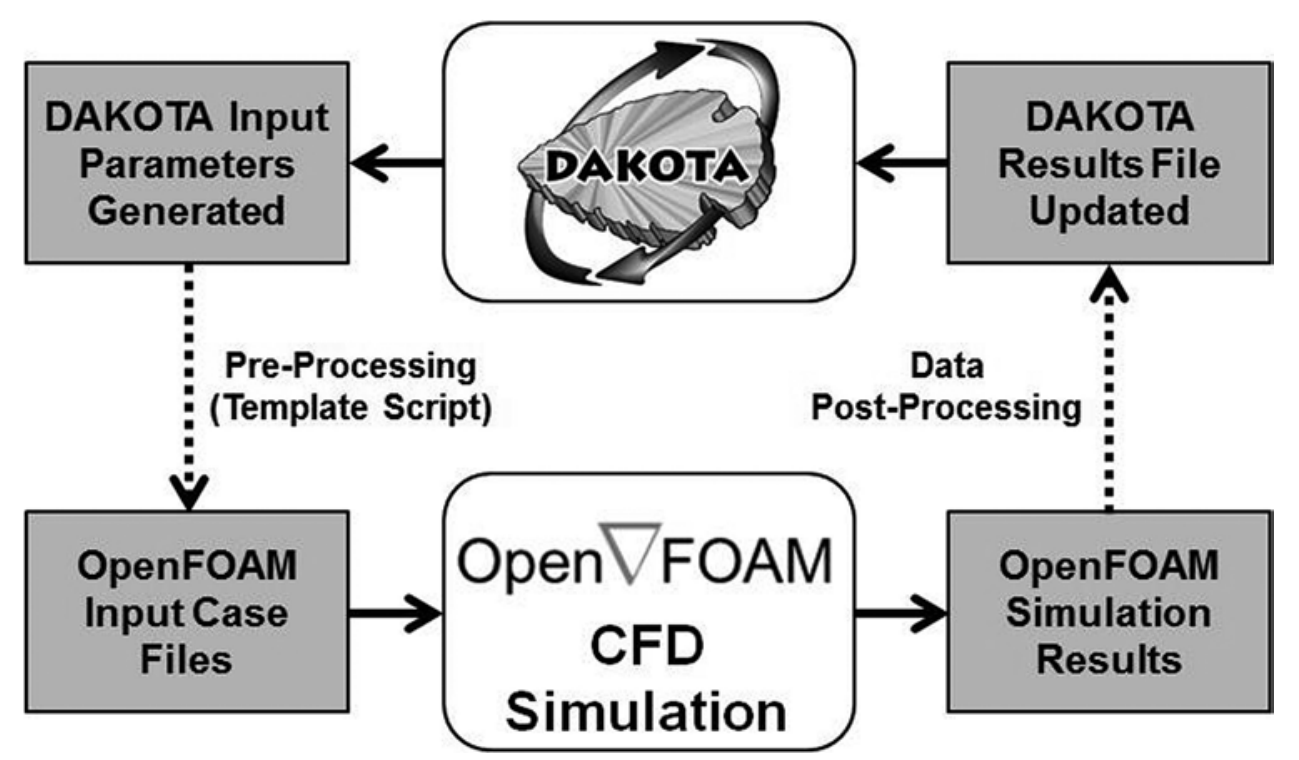

FIG. 1. Schematic representation of the framework that couples the DAKOTA optimization software to the OpenFOAM CFD software. 
Ltd.) performs the CFD simulation and includes utilities for building the computational domain and mesh, numerically computing the flow fields, and extracting relevant postanalysis data. OpenFOAM can be controlled completely via user-written scripts, a requirement for design exploration using automated execution.

The Design Analysis Kit for Optimization and Terascale Applications (DAKOTA, Release 6.2, Sandia Corporation) serves as the parameter manager, supplying geometrical inputs to OpenFOAM, collecting the processed outputs from each simulation, and then methodically selecting the next set of geometrical inputs to be tested. Given an objective function, DAKOTA can then iteratively optimize a set of input parameters toward a specific goal. In the demonstration example, the goal is uniform inlet flow rate distribution, and the inputs are the catheter's geometrical parameters. Flow rate distribution in the demonstration is characterized by the standard deviation of the inlet hole flow rates, which serves as the objective function that must be minimized by DAKOTA.

For a given catheter configuration (e.g., rounded or elongated tip, parallel or staggered rows of inlet holes, etc.) and boundary conditions (e.g., communicating or noncommunicating hydrocephalus, type of shunt valve, etc.), a baseline model made up of several script files is generated using OpenFOAM's basic case structure. OpenFOAM's blockMesh utility was chosen to model the fluid domain and catheter geometry because it allows direct user control of all meshing parameters. This utility is controlled via a template script that enables automated domain and mesh generation for a set of uploaded geometrical parameters. This template will change, depending on the given baseline catheter architecture. Once implemented, with each new set of uploaded parameters, a new catheter model is automatically generated and the fluid domain is meshed in preparation for computational evaluation of the model.

This allows for reliable and reproducible automated CFD simulations, performed using OpenFOAM. For the incompressible, steady-state simulation of a Newtonian fluid used for this model, the simpleFoam solver was selected for the numerical solution of the Navier-Stokes equations of fluid flow. Using this solver, the fluid pressure and velocity values are numerically approximated at each node of the mesh, and the volumetric flux, or volumetric flow rate, is also calculated for each finite volume face in the mesh. Depending on the objective of the optimization, any one of these values can be monitored and sent to DAKOTA as an output of the CFD simulation. In the demonstration example, the flow rates of each of the inlet holes are monitored and a standard deviation of flow rates is calculated to rate the uniformity of the catheter's inlet flow rate profile for each specific geometrical variation.

DAKOTA offers many tools to explore parametric effects on an objective function. Depending on the optimization methods chosen, the outputs sent to DAKOTA from the completed OpenFOAM simulation will be recorded and a new set of input parameters will be sent for model generation and simulation. This iterative series of simulations will continue until the criteria of the optimization or parametric sensitivity study are satisfied.

\section{Demonstration Example}

The computational framework was demonstrated by optimizing a typical, commercially available baseline catheter architecture to produce a uniform flow rate distribution across the catheter's inlet holes, the objective function identified in previous studies by Lin et al..$^{20}$ and Galarza et al ${ }^{7-10}$ This problem was chosen given its importance in the literature and the opportunity to compare the optimized design calculated by the framework with the results of other researchers. A baseline 3D CFD model was developed for a ventricular catheter within a cylindrical ventricular chamber (Fig. 2), and the geometrical parameters of the catheter were systematically varied to investigate their influence on the catheter's fluid performance.

Investigation of this design objective began with the landmark study of Lin et al. ${ }^{20}$ which computationally and experimentally explored the flow profile of commercially available catheters. Using 2D CFD simulation, this study demonstrated that the majority of CSF flows at high flow rates into the proximal holes (those farthest from the catheter tip, Fig. 3), while the distal holes (those closest to the tip) were ineffective, with extremely low flow rates. Under these flow conditions, it was concluded that obstructions are far more likely to enter the catheter through a proximal

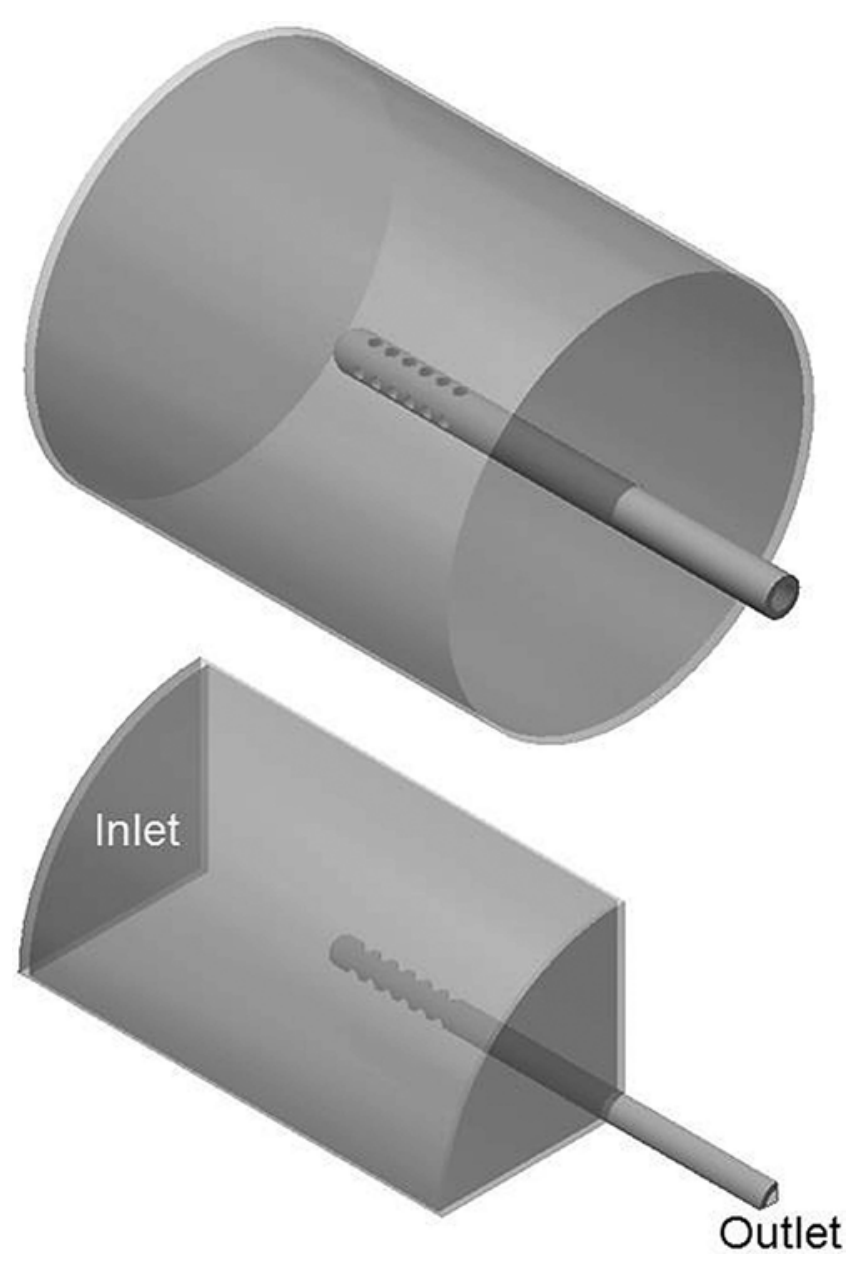

FIG. 2. Concept for the 3D computational model. The reduced quartersection domain is shown with inlet and outlet identified. 


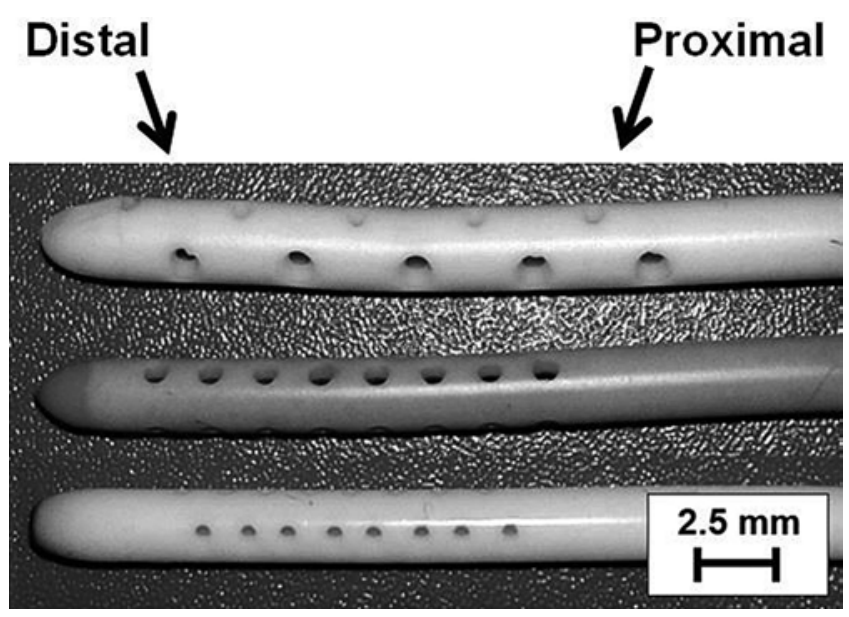

FIG. 3. Photograph showing 3 different commercial styles of catheter tips with varying inlet hole configurations. Distal and proximal hole sets are identified.

hole, potentially blocking the flow entirely and rendering the distal holes useless.

Explanted occluded ventricular catheters provided further evidence to support this conclusion, as occlusions were observed to occur most often at the proximal set of holes. ${ }^{20}$ These observations led to the hypothesis that a catheter geometry that results in a more uniform flow rate distribution among the catheter's inlet holes will reduce the statistical likelihood of an obstruction occurring at the critical proximal inlet holes, thereby reducing shunt failure rates due to ventricular catheter obstruction. As a result of this research, the Rivulet (Medtronic Neurosurgery) catheter was developed with a design consisting of decreasing hole diameters from the distal to proximal end (https:// www.accessdata.fda.gov/cdrh_docs/pdf6/K063836.pdf). CFD simulation ${ }^{10}$ has shown this design to be effective at producing a more uniform flow distribution, although no clinical studies to date have proven its effectiveness at reducing obstruction rates. Continuing the effort to develop a catheter that achieves such a uniform flow profile, Galarza et al. pioneered the use of 3D CFD to further investigate the effects of geometry variation on CSF flow profiles. ${ }^{7-10}$

Some geometrical parameters of significance had been identified in previous studies, and their investigation informed the selection of parameters for this demonstration. Galarza et al. ${ }^{8}$ showed that a decreasing intersegment distance ( the distance between sets of holes along the length of the catheter) and a decreasing hole diameter distribution, initiating at the distal end, as well as a small number of holed segments are highly influential in obtaining a uniform flow profile. The same study also demonstrated that the number of holes at the proximal segment, relative rotations of the drainage segments around the axis of the catheter, and the addition of a patent hole at the catheter tip do not significantly influence the flow pattern. Thomale et al. ${ }^{27}$ investigated the effect of the total number of holes on obstruction rates for the specific case of catheters placed in narrow ventricles, determining that catheters with substantially fewer holes, but possessing an equal flow distribution, could decrease obstruction, provided the catheter was properly placed. In addition to investigating geometry that results in a uniform flow distribution, Harris and McAllister ${ }^{15}$ and Ginsberg et al. ${ }^{11}$ also correlated increased shear stress along the catheter/CSF interface to an increased likelihood of occlusion; and Cheatle et al. ${ }^{3}$ determined that the fluid resistance of new and explanted catheters is related to the diameter, number of holes, and age of the catheter.

These studies influenced the selection of a baseline model as well as the parameters to be investigated. The baseline catheter configuration used for this demonstration of the framework consisted of 4 parallel rows of inlet holes, equally spaced around the catheter's circumference, and each row had 6 holes. The number of holes, parallel row configuration, and catheter internal diameter of 1.5 $\mathrm{mm}$ were held fixed while other geometrical parameters were varied: catheter wall thickness, distance from catheter tip to the first inlet hole, spacing distances between inlet holes, inlet hole diameters, and the degree of tapering of the inlet holes (Fig. 4). First, the sensitivity of each parameter on the objective function was quantified, and then the design was optimized using the most influential parameters identified in the sensitivity analysis. It should be noted that, in general, the framework will operate on a parametric model for any baseline configuration, and the number of holes, hole configuration, and internal diameter can also be specified as variables. More complex fluid dynamics and variable boundary conditions can also be investigated using this framework.

\section{CFD Model Development}

Prior work by Lin et al..$^{20}$ and Galarza et al. ${ }^{7-10}$ forms the foundation for the computational investigation of CSF flow through ventricular catheters. The CFD model developed for this demonstration follows similar boundary conditions and assumptions with one significant difference: the catheter's outlet boundary conditions. To model obstructive (noncommunicating) hydrocephalus, the catheter is placed in the center of an enlarged CSF-filled ventricle that is represented as a rigid, fixed volume, cylindrical chamber (Fig. 3). The catheter outlet is located outside the walls of the chamber, reflecting the in vivo placement of most ventricular catheters. The catheter provides the only outlet for CSF exiting the ventricle, and the incoming flow into the chamber is from the slow, steady production of CSF within the ventricle. Since CSF is composed almost completely of water ${ }^{16}$ the fluid properties used for the model were those of water at a body temperature of $37^{\circ} \mathrm{C}$.

A constant exit flow rate of $0.35 \mathrm{ml} / \mathrm{min}$ was used as the main driving boundary condition to mimic the average flow rate induced by natural CSF circulation or by a valveless shunt. ${ }^{25}$ In addition, a constant zero pressure was defined at the inlet (Fig. 3), and a no-slip boundary condition was defined at the catheter walls and at the solid domain boundaries. Fluid-structure interactions between the CSF and the catheter were not included in this simulation, as the fluid flow is very slow and is therefore not expected to substantially influence the catheter geometry. The simulations were run as steady state and thus do not account for any pulsatile effects. Since the flow remains laminar during the whole pulse, a quasi-steady flow behavior is expected (i.e., the flow pattern remains the same, but the 


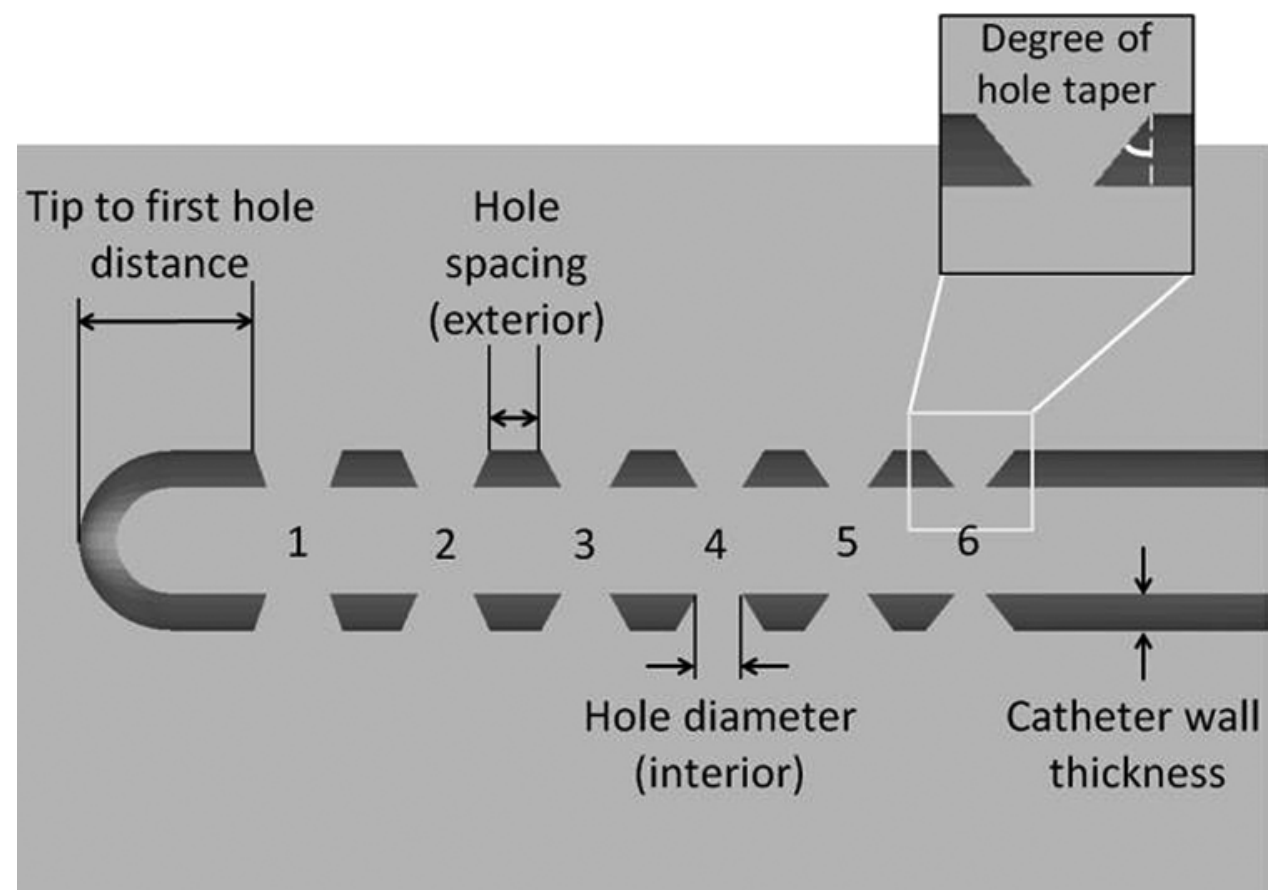

FIG. 4. Schematic representation of ventricular catheter model cross-section with parameters of interest labeled. Inlet holes are numbered starting from those closest to the catheter tip (distal end).

velocity scales accordingly). However, a calculated Womersley number greater than 1 could indicate that the flow through ventricular catheters may be moderately affected by CSF pulsation within the ventricles. This behavior could be investigated using the computational framework, given a validated baseline model that includes this effect.

To isolate the effects of catheter geometry from the effects of ventricle shape, the fluid domain around the catheter was sized to minimize wall effects. Because computational time increases with the domain size, modeling the smallest domain without inducing significant wall effects was essential when considering the massive number of analyses required for optimization. The results of a convergence study indicated that a distance of $6 \mathrm{~mm}$ between the chamber walls and the catheter outer wall, first inlet hole, and last inlet hole was the smallest domain size to minimize wall effects. This value was held constant across all simulations.

The laminar flow conditions and parallel 4-row configuration of the catheter enabled the use of a quarter model to characterize the flow field while greatly reducing the computational domain (Fig. 3). To achieve high accuracy in areas of interest while minimizing computation time, finer grids were specified around the catheter inlet holes, with coarser grids around the borders of the cylindrical chamber (Fig. 5). A mesh refinement study determined that an average of 350,000 finite volume elements were required to reach the convergence criteria for this baseline model and to limit convergence time to allow for efficient automated simulation iterations.

In this example, the goal is uniform inlet flow rate distribution and the inputs are the catheter's geometrical parameters. Flow rate distribution is characterized by the standard deviation of the inlet hole flow rates, which serves as the objective function that must be minimized by DAKOTA. During the OpenFOAM analysis, the flow rates of each of the inlet holes are monitored for convergence, and a standard deviation of flow rates is calculated to rate the uniformity of the catheter's inlet flow rate profile for each specific geometrical variation. This metric was the summary output sent to DAKOTA during subsequent iterative analyses and served as a basis for comparing the various tested designs.

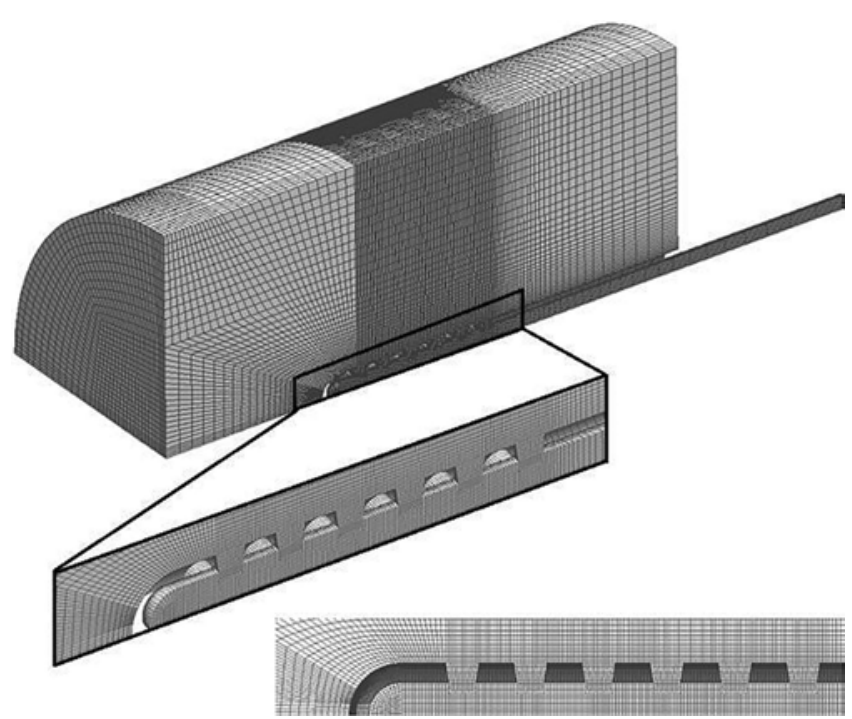

FIG. 5. Example of a meshed computational fluid domain. The close-up views show the area of interest around the catheter's inlet holes using a highly refined mesh. 


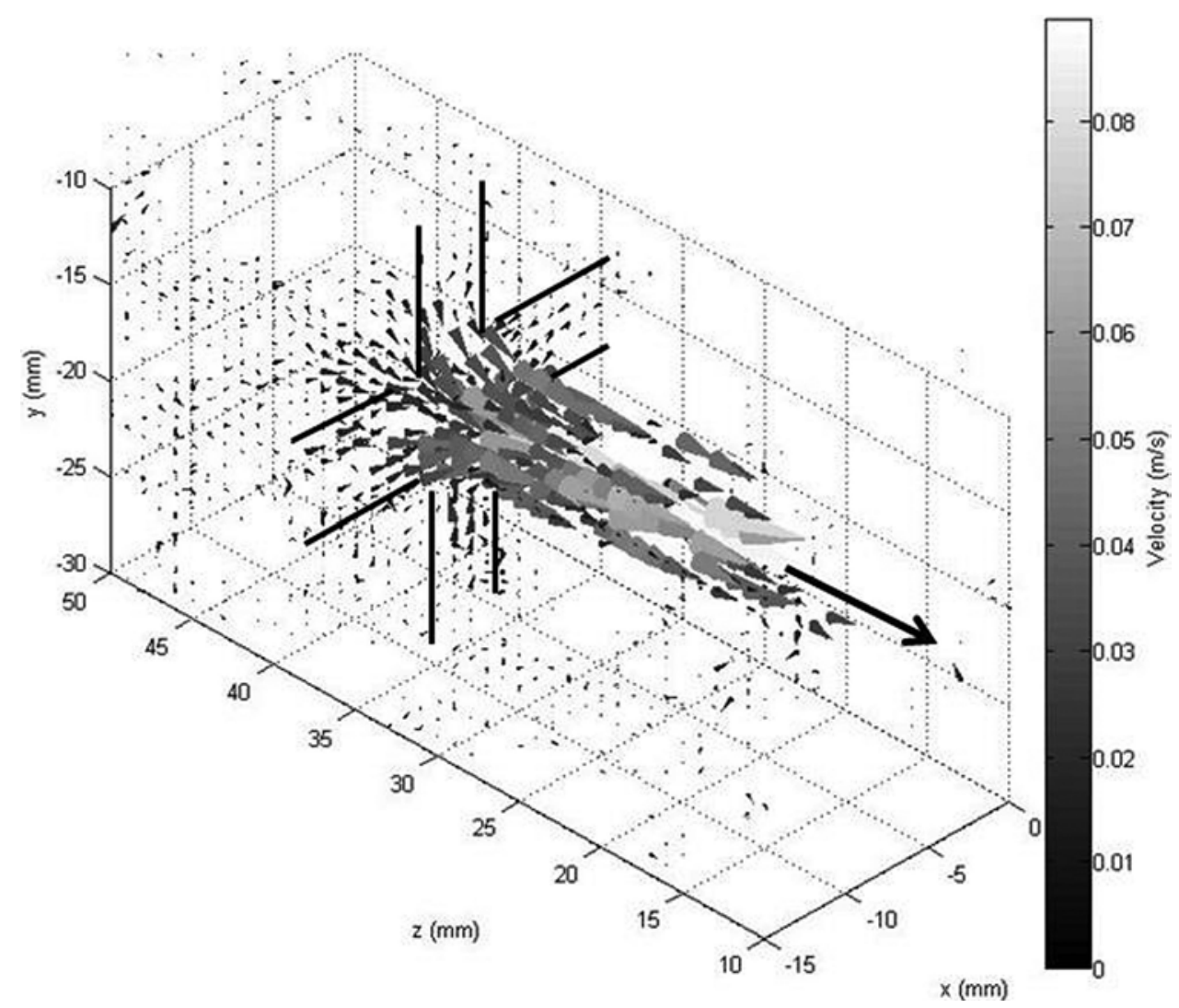

FIG. 6. Velocity plot of 3D fluid imaging through a scaled-up catheter model using PEPT, showing a close-up around the proximal hole set. Black boundaries are overlaid to show proximal hole locations and the black arrow shows direction of flow. $s=$ second.

\section{Validation of CFD Model Using Positron Emission Particle Tracking}

Before performing iterative CFD analyses using the computational framework, the baseline CFD model was experimentally validated. Nuclear imaging using positron emission particle tracking (PEPT) was performed to visualize the 3D flow field around and through a catheter and to provide quantified data to validate the flow rates obtained using the baseline CFD model. Performed in a positron emission tomography (PET) scanner, this nuclear imaging method tracks particles laden with a radionuclide tracer as they flow through a given test section. ${ }^{21}$ In this validation test, a scaled-up version of a standard 32-hole catheter with a parallel 4-row configuration was used. Scaling the catheter was necessary because the resolution of the scanner would not have been sufficient to image the flow to the inlet holes of a commercial catheter. Therefore, a catheter was machined out of clear polyethylene terephthalate glycol-modified plastic (PETG) tubing with inner diameter of 0.25 inches (approximately 4.5 times larger than a standard commercial catheter). Flow properties were modified, using Reynolds' scaling principles, to match the flow regimen simulated by the computational model.

The catheter was immersed in the particle solution and connected to a pump, causing flow to enter through the catheter's inlet holes. Approximately 1600 particle trajectories were collected as the particles moved through the imaged fluid section. Postprocessing of these trajectories created a 3D velocity flow field (Fig. 6), indicating the average velocities of the tracked particles at specific locations. As a point of comparison, the flow rates at the proximal inlet holes were examined. In the scaled-up test and an unscaled simulation using the proposed CFD model, the flow rates at the proximal inlet holes comprised 56.8\% and $56.06 \%$ of the total incoming flow, respectively. These results also match well with the results of the 2D CFD study performed by Lin et al., in which $58 \%$ of the total flow enters through the proximal hole set in this catheter design. The simulated incoming velocity at this hole set also matched the experimental results with similar accuracy. The flow rates of more distal inlet holes were more difficult to compare using this method because the number of particles entering through them was relatively small and therefore could not provide a statistically robust average velocity through those holes.

Previous studies used 2D methods, ${ }^{20} 3 \mathrm{D}$ ink visualization, $, 8,20,27$ and evaluation of pressure/flow characteristics ${ }^{4}$ to experimentally investigate CSF flow through a catheter. In this initial test, 3D nuclear imaging exhibited potential as a viable method to capture quantified fluid velocities at specific locations along the catheter flow trajectory.

\section{Parametric Sensitivity Studies}

The validated CFD model served as the baseline for exploration and optimization of geometrical modifications relative to the objective function of uniform inlet hole flow 
TABLE 1. One-at-a-time parameter sensitivity study results

\begin{tabular}{|c|c|c|c|c|}
\hline \multirow[b]{2}{*}{ Parameter } & \multicolumn{2}{|c|}{ Range } & \multirow[b]{2}{*}{ Considerations } & \multirow{2}{*}{$\begin{array}{l}\text { Sensitivity Index } \\
\text { (for given range) }\end{array}$} \\
\hline & Min & Max & & \\
\hline Inlet hole diameter (uniform) & $0.25 \mathrm{~mm}$ & Catheter ID & Structural integrity, preventing large obstructions & 0.9589 \\
\hline $\begin{array}{l}\text { Linear factor for hole diam- } \\
\text { eters }\end{array}$ & $0.90 \mathrm{~mm}$ & $1.10 \mathrm{~mm}$ & $\begin{array}{l}\text { Representative sampling, structural integrity, max length of perfo- } \\
\text { rated catheter segment }\end{array}$ & 0.9054 \\
\hline Inlet hole degree of tapering & $0^{\circ}$ & $16^{\circ}$ & Fabrication process, sample values extracted from previous study & 0.9028 \\
\hline Catheter wall thickness & $0.45 \mathrm{~mm}$ & $0.80 \mathrm{~mm}$ & Structural integrity, PDMS extrusion limitations & 0.3620 \\
\hline $\begin{array}{l}\text { Hole spacing distance } \\
\text { (uniform) }\end{array}$ & Wall thickness & $2.00 \mathrm{~mm}$ & Structural integrity, max length of perforated catheter segment & 0.3605 \\
\hline $\begin{array}{l}\text { Linear factor for hole spacing } \\
\text { distance }\end{array}$ & $0.90 \mathrm{~mm}$ & $1.10 \mathrm{~mm}$ & $\begin{array}{l}\text { Representative sampling, structural integrity, max length of perfo- } \\
\text { rated catheter segment }\end{array}$ & 0.2460 \\
\hline $\begin{array}{l}\text { Distance from tip to first inlet } \\
\text { hole }\end{array}$ & $\begin{array}{l}\text { Catheter OD + } \\
\text { wall thickness }\end{array}$ & $5.00 \mathrm{~mm}$ & Rounded tip radius, max length of perforated catheter segment & 0.0003 \\
\hline
\end{tabular}

$\mathrm{ID}=$ inner diameter; $\mathrm{OD}=$ outer diameter.

Modified from a table that first appeared in the academic thesis Improving Ventricular Catheter Design Through Computational Fluid Dynamics (Weisenberg SH,

University of Tennessee, Knoxville, 2016).

rate distribution. For this objective function, the standard deviation of the relative inlet hole flow rates quantified the performance of each catheter design variation. A higher value indicated a more skewed relative flow rate profile, while a zero value represented complete uniformity among the inlet hole flow rates. Therefore, the objective function was specified as minimizing this output of the CFD simulations.

To initialize design optimization, a sensitivity analysis was performed to systematically explore the effects of geometry modifications on the flow profile, and a subsequent investigation of the parameter space determined the initial conditions required for the local optimization method. Table 1 lists the geometrical modifications that were evaluated using a one-factor-at-a-time approach. Each parameter was varied independently to quantify its influence on the objective function, and a sensitivity index was computed. A higher sensitivity index indicates a higher influence, and the index is independent of the parameter's units but dependent on the specified range for testing.

Parameter ranges for the study were determined based on maintaining the catheter's structural integrity and followed fundamental engineering principles. Inlet hole diameters were characterized by the diameter measured at the interior lumen of the catheter, and the maximum value was defined as the diameter at which the inlet holes, spaced $90^{\circ}$ apart around the circumference of catheter, would begin to intersect at the interior catheter lumen. Hole spacing along the length of the catheter was measured along the exterior surface, and a minimum value of 1.5 times the wall thickness was specified to preserve the structural viability of the catheter. The total length of the perforated segment is commonly limited to the $1.5 \mathrm{~cm}$ closest to the catheter tip, to increase the probability that the inlet holes remain inside the ventricle after surgical placement. This was also taken into account as a limiting factor in choosing parameter ranges.

To reduce the number of individual parameters tested in this initial study, the hole diameters and hole spacing were manipulated in 2 separate ways: 1) 1 variable value was chosen and uniformly designated for all holes in the catheter, or 2) 1 value was chosen for the most distal hole and a variable linear factor was applied to calculate the values for the subsequent holes. The linear factor method allowed investigation of the effect of linearly decreasing or increasing hole diameters or spacing on the objective function.

Hole tapering was varied as a uniform parameter for all holes. Tapering is usually a byproduct of the hole punching process. Most manufacturers strive for a straight bore without taper, presumably to limit possible obstructions that could accumulate at a tapered hole. However, during hole punching, compressive and tensile stresses cause distortions at either end of the hole, and after the material undergoes relaxation, a tapered hole is created. To estimate a range for possible tapers, photographs of punched sample catheters, from a study demonstrating the effects of catheter hole size on cellular adhesion, ${ }^{14}$ were examined to estimate common degrees of tapering.

The following conclusions were drawn from the sensitivity analysis:

- The diameters of the holes are the most influential parameters; both the diameter value of the first hole and subsequent applications of a linear factor to the remaining holes caused significant changes in the objective function.

- Linear scaling factors of less than 1 for the hole diameters produced favorable objective functions, confirming past studies showing that designs featuring decreasing hole diameters improve flow rate uniformity.

- Linear factors between 1.0 and 1.1 showed the most promise with regard to hole spacing, indicating that, moving away from the tip, increasing spaces between the holes would produce favorable results. However, the sensitivity index for this parameter was relatively low, so its influence was not as substantial as that of other parameters.

- The degree of hole tapering was significantly influential, and although this parameter is not easily modified in catheter manufacturing, it is apparent that some taper- 
ing $\left(5^{\circ}-10^{\circ}\right)$ of the holes is beneficial in terms of improving flow rate uniformity.

- Wall thickness was less influential, but lower objective function values were observed for thicker catheter walls.

- The distance from the catheter tip to Hole 1 was the least influential parameter, with a sensitivity index several scales of magnitude less than those of the other parameters.

\section{Design Optimization}

The conclusions from the sensitivity study provided a basis for excluding parameters that exhibited minimal influence from the subsequent and more comprehensive optimization investigation. This sensitivity analysis was repeated using similar baseline models with 4 and 8 holes per row, also typical in commercially available configurations, and the parameter rankings of influence remained consistent. A standard distance from the catheter tip to Hole 1 was selected as $1.2 \mathrm{~mm}$, since this parameter proved the least influential. The low influence of this parameter can be attributed to the fact that this region sees little to no flow. Moderately influential factors, such as degree of hole tapering and wall thickness, were evaluated at only 1 or 2 values. For degree of hole tapering, the value of $7.688^{\circ}$ was held constant since this value was shown to minimize the objective function in the 6-hole sensitivity study. For wall thickness, standard thicknesses of extruded silicone tubing of $0.5 \mathrm{~mm}$ or $0.6 \mathrm{~mm}$ were evaluated. Since the material properties of the catheter (flexibility, structural integrity) depend on this factor, it was important not to include significantly thicker or thinner catheters, as these may not be viable for use in vivo. With these parameters limited (or set), the remaining parameters were carried forward for optimization.

Next, Latin hypercube sampling (LHS) was employed for sampling the parameter space created by only varying the most influential parameters. This method generated a collection of input parameter sets that effectively covered the entire space. Several thousand sample simulations were performed for the exploration of this space, which includes the effects of parameter interactions, a complexity not accounted for in the one-factor-at-a-time study. By investigating the results of this sampling, several areas of interest, where the objective function was minimized, were located within the parameter space. Initial points, selected from these areas of interest, were used to initialize the more in-depth local optimization method.

A local, derivative-free optimization algorithm (COLINY Pattern Search) was selected in DAKOTA to supply the framework with geometrical input parameters and identify the optimal set. As with all local optimization methods, the outcome is dependent on the initial points supplied to the search algorithm, and it is possible to calculate a different optimized geometry for each set of initial parameters chosen. Therefore, identifying initial points based on both sensitivity studies and general parameter space sampling was critical to the success of this method. Moreover, a dimensional analysis for this fluid model shows that the number of degrees of freedom in this study also preclude the existence of a unique solution for this optimization objective. After several hundred evaluations using this optimization algorithm, the geometrical parameter set that most minimized the objective function was recorded and is presented as the optimized design.

Parallel processing provided the computational efficiency required for such a comprehensive investigation of catheter design. In this demonstration example, the computational framework was implemented on the Darter supercomputer (a Cray XC30 system) located at the National Institute for Computational Sciences on the Oak Ridge National Laboratory campus (https://www.nics.tennessee. edu/computing-resources/darter). Parameter exploration and optimization processes were executed in parallel on 20 compute nodes (320 processors). Using this system, the analysis time for CFD simulation of 1000 catheter models was approximately 30 minutes, and the entire optimization process required approximately 5 hours of computational analysis.

\section{Results}

After running the optimization analysis several times, the catheter geometry yielding the lowest objective function value was selected. The high-fidelity OpenFOAM simulation results of this catheter design are shown in Fig. 7 , and the final set of geometrical parameters is presented in Table 2. This catheter design featured decreasing hole diameters, moving away from the catheter tip; this validates the results of previous studies that showed the promise of such designs..$^{10,20}$ It also featured slightly increasing hole spacing distances, moving away from the catheter tip, for a total perforated segment length of approximately $10.5 \mathrm{~mm}$, which is within the normal range for most ventricular catheters currently on the market.

The optimization strategy employed was effective in the identification of local minima in the response function, when supplied with a favorable initial point. The results of the design optimization demonstrated a drastic improvement over a "standard" catheter design featuring uniform hole diameters and spacing. As seen in Fig. 7, the standard design had a computed objective function of $14.27 \%$, an obviously flawed flow profile compared with the optimized result of $0.30 \%$, which demonstrates nearly complete uniformity of flow rates between the inlet holes. Even previous CFD analysis of the Medtronic Rivulet catheter design shows a standard deviation of approximately $4.5 \%$ among the relative inlet hole flow rates..$^{10}$ As previously stated, this method of optimization could theoretically locate other such favorable sets of geometrical parameters, making the design presented here not unique in its ability to improve the inlet flow rate profile but certainly a good example of the capabilities of this computational framework.

\section{Discussion}

The results of this example demonstrate the capabilities of this computational framework to significantly improve ventricular catheter design. Sensitivity analysis, used to identify the most influential input parameters, overcomes a major limitation in optimization by eliminating less influential parameters, thereby reducing the dimensions of 


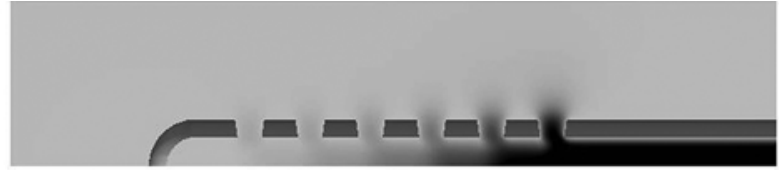

Velocity Magnitude (m/s)
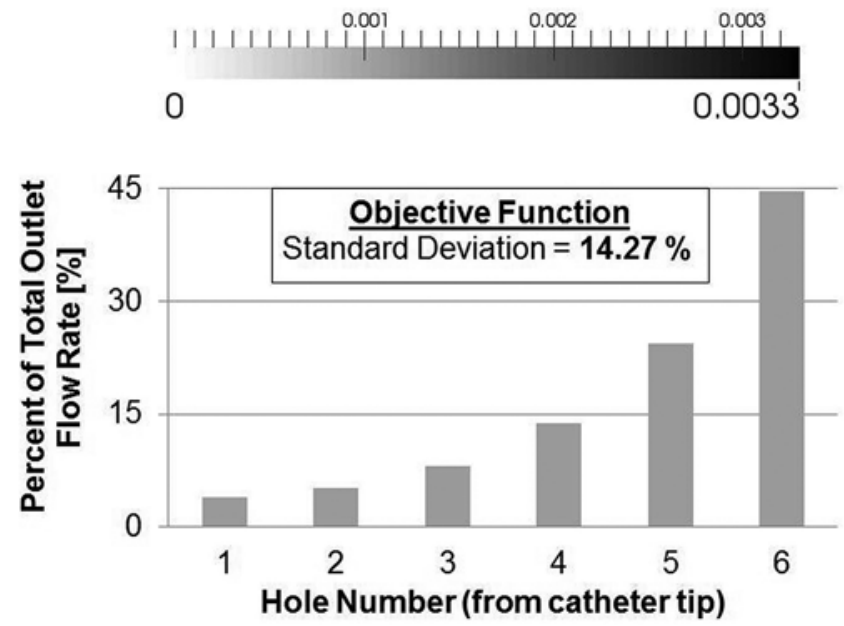

FIG. 7. Comparison of optimized catheter design (right) and common commercially available catheter with uniform hole diameters and spacing (left). The upper plots show visualization of fluid velocity magnitude in a cross-section of the catheter. The lower plots show the distribution of relative flow rates among the catheter's inlet holes. The objective function is specified for each design.

the parameter space. Additionally, a supercomputing environment enables analysis of high-fidelity models of complex scenarios representing the in vivo performance of the shunt system in a feasible amount of computation time. Such models could readily include more physically realistic details, such as varying ventricle size and shape, pulsating fluid flow, and transient flow induced by the valve opening as well as unique geometrical designs with varying numbers of holes and hole configurations.

For example, to include the effects of a shunt valve opening, the CFD analysis would include transient simulations (changing over time) rather than the steady-state simulations used in this current study. The inlet hole flow rates would change over time and the efficacy of each geometrical configuration would be evaluated and compared over each time step within a given cycle of CSF pulsation or valve operation. While adding such behaviors or including turbulent effects in the baseline model would significantly increase the computational time required to evaluate each new set of geometrical inputs, the insights gained could be extremely valuable in understanding the impact of the sometimes erratic flow within ventricular catheters on flow uniformity through the catheter holes. Therefore, more advanced sensitivity analysis methods, including parameter interactions and higher-order effects, should be further explored as a new, automated module in the framework. Advanced sensitivity analysis to identify the most influential parameters and parameter interactions would inform identification of parameter limited subspaces that could then be optimized for complex models in a feasible timeframe.

Any baseline catheter design can be parametrized and investigated using the computational framework described here. For example, staggered row designs, which are also popular among the leading catheter manufacturers, may add vortices or other flow phenomena that could significantly influence the fluid flow performance of these catheters. Additionally, the number of holes may be a parameter of interest to test in future systematic simulation studies, as previous publications have shown similar effectiveness for ventricular catheters bearing only a small number of inlet holes. ${ }^{11,27}$

New input templates following the same development procedure can be created for emerging designs, and objective functions for additional flow characteristics that are identified to influence obstruction rates, such as wall shear stress, can be included in the optimization. Furthermore, moving in the direction of personalized medicine, a patient's unique condition can be addressed by changing a few boundary conditions and optimizing catheter design to fit specific patient needs and limitations; for example, the age and size of the patient, the measured intracranial pressure, or more uncommon conditions such as slit-ventricle syndrome or normal pressure hydrocephalus.

Validation of CFD models is also critical to ensure that the simulation is a physically realistic representation. Quantification of in vivo conditions would enable more complex boundary conditions in the models, and enhanced knowledge of fluid dynamics into and through the catheter is essential to better understanding the flow profiles that will reduce the likelihood of obstruction. PEPT imaging is a viable technique to generate quantified 3D flow data, but it is limited by the number of particles, the need to recirculate the particles, the radioactive decay time frame of the particles, and the size of the particles. While scaling up the catheter eliminates some of these limita- 
TABLE 2. Final set of geometric parameters for optimized ventricular catheter design

\begin{tabular}{|c|c|}
\hline Parameter & Value \\
\hline Catheter ID (mm) & 1.50 \\
\hline Wall thickness (mm) & 0.60 \\
\hline Degree of hole tapering & $7.688^{\circ}$ \\
\hline Total length of perforated segment (mm) & 10.495 \\
\hline \multicolumn{2}{|l|}{ Interior hole diameter (mm) } \\
\hline ID 1 & 0.6876 \\
\hline ID 2 & 0.6112 \\
\hline ID 3 & 0.5254 \\
\hline ID 4 & 0.4560 \\
\hline ID 5 & 0.4030 \\
\hline ID 6 & 0.3616 \\
\hline \multicolumn{2}{|l|}{ Exterior hole diameter (mm) } \\
\hline OD 1 & 0.8496 \\
\hline OD 2 & 0.7732 \\
\hline OD 3 & 0.6874 \\
\hline OD 4 & 0.6180 \\
\hline OD 5 & 0.5650 \\
\hline OD 6 & 0.5236 \\
\hline \multicolumn{2}{|l|}{ Hole spacing distance (mm) } \\
\hline SP: Tip to Hole 1 & 1.2000 \\
\hline SP: Hole 1 to Hole 2 & 1.0000 \\
\hline SP: Hole 2 to Hole 3 & 1.0750 \\
\hline SP: Hole 3 to Hole 4 & 1.1556 \\
\hline SP: Hole 4 to Hole 5 & 1.2423 \\
\hline SP: Hole 5 to Hole 6 & 1.3355 \\
\hline
\end{tabular}

$\mathrm{SP}=$ spacing .

Modified from a table that first appeared in the academic thesis Improving Ventricular Catheter Design Through Computational Fluid Dynamics (Weisenberg SH, University of Tennessee, Knoxville, 2016).

tions, testing with a much larger catheter representation may not produce realistic data when evaluating the potential for obstructions. Alternative experimental procedures, such as particle image velocimetry, should continue to be developed to provide capabilities to fully characterize and quantify 3D CSF flow behavior.

\section{Conclusions}

This computational framework for design exploration and improvement is an efficient and highly customizable tool to rapidly explore the effects of geometry modifications on CSF flow into and through ventricular catheters. This approach overcomes the time limitation of manually creating new catheter models for each design modification and allows the analysis of thousands of designs in a matter of hours, as was demonstrated for the baseline model evaluated in this study.

This framework should represent part of a larger effort to improve the computational tools available to both engineers and surgeons working with CSF shunts. More comprehensive shunt models that include complex fluid dynamics behavior would offer invaluable insights that are currently only available through clinical testing of prototypes or highly simplified bench testing. Computational simulation can strengthen our currently inadequate understanding of in vivo shunt performance and our ability to prevent ventricular catheter obstruction. This knowledge is essential to reducing the number of revision surgeries and ultimately improving the life quality of hydrocephalus patients.

\section{References}

1. Appelgren T, Zetterstrand S, Elfversson J, Nilsson D: Longterm outcome after treatment of hydrocephalus in children. Pediatr Neurosurg 46:221-226, 2010

2. Browd SR, Ragel BT, Gottfried ON, Kestle JRW: Failure of cerebrospinal fluid shunts: part I: Obstruction and mechanical failure. Pediatr Neurol 34:83-92, 2006

3. Cheatle JT, Bowder AN, Agrawal SK, Sather MD, Hellbusch LC: Flow characteristics of cerebrospinal fluid shunt tubing. J Neurosurg Pediatr 9:191-197, 2012

4. Czosnyka M, Czosnyka Z, Momjian S, Pickard JD: Cerebrospinal fluid dynamics. Physiol Meas 25:R51-R76, 2004

5. Drake JM, Kestle JR, Milner R, Cinalli G, Boop F, Piatt J Jr, et al: Randomized trial of cerebrospinal fluid shunt valve design in pediatric hydrocephalus. Neurosurgery 43:294-305, 1998

6. Duncan CC: Management of proximal shunt obstruction. Technical note. J Neurosurg 68:817-819, 1988

7. Galarza M, Giménez Á, Pellicer O, Valero J, Amigó JM: New designs of ventricular catheters for hydrocephalus by 3-D computational fluid dynamics. Childs Nerv Syst 31:3748, 2015

8. Galarza M, Giménez A, Pellicer O, Valero J, Amigó JM: Parametric study of ventricular catheters for hydrocephalus. Acta Neurochir (Wien) 158:109-116, 2016

9. Galarza M, Giménez Á, Valero J, Pellicer O, Martínez-Lage JF, Amigó JM: Basic cerebrospinal fluid flow patterns in ventricular catheters prototypes. Childs Nerv Syst 31:873-884, 2015

10. Galarza M, Giménez Á, Valero J, Pellicer OP, Amigó JM: Computational fluid dynamics of ventricular catheters used for the treatment of hydrocephalus: a 3D analysis. Childs Nerv Syst 30:105-116, 2014

11. Ginsberg HJ, Sum A, Drake JM, Cobbold RS: Ventriculoperitoneal shunt flow dependency on the number of patent holes in a ventricular catheter. Pediatr Neurosurg 33:7-11, 2000

12. Go KG, Ebels EJ, van Woerden H: Experiences with recurring ventricular catheter obstructions. Clin Neurol Neurosurg 83:47-56, 1981

13. Hakim S: Observations on the physiopathology of the CSF pulse and prevention of ventricular catheter obstruction in valve shunts. Dev Med Child Neurol Suppl 20:42-48, 1969

14. Harris CA, McAllister JP II: Does drainage hole size influence adhesion on ventricular catheters? Childs Nerv Syst 27:1221-1232, 2011

15. Harris CA, McAllister JP II: What we should know about the cellular and tissue response causing catheter obstruction in the treatment of hydrocephalus. Neurosurgery 70:15891602, 2012

16. Irani DN: Cerebrospinal Fluid in Clinical Practice. Philadelphia: Saunders Elsevier, 2009

17. Kehler U, Langer N, Gliemroth J, Meier U, Lemcke J, Sprung $\mathrm{C}$, et al: Reduction of shunt obstructions by using a peel-away sheath technique? A multicenter prospective randomized trial. Clin Neurol Neurosurg 114:381-384, 2012

18. Kestle J, Drake J, Milner R, Sainte-Rose C, Cinalli G, Boop F, et al: Long-term follow-up data from the Shunt Design Trial. Pediatr Neurosurg 33:230-236, 2000 
19. Kossovsky N, Snow RB: Clinical-pathological analysis of failed central nervous system fluid shunts. J Biomed Mater Res 23 (A1 Suppl):73-86, 1989

20. Lin J, Morris M, Olivero W, Boop F, Sanford RA: Computational and experimental study of proximal flow in ventricular catheters. Technical note. J Neurosurg 99:426-431, 2003

21. Ruggles AE, Zhang BY, Peters SM: Positron emission tomography (PET) for flow measurement. Adv Mat Res 301303:1316-1321, 2011

22. Sainte-Rose C: Shunt obstruction: a preventable complication? Pediatr Neurosurg 19:156-164, 1993

23. Sekhar LN, Moossy J, Guthkelch AN: Malfunctioning ventriculoperitoneal shunts. Clinical and pathological features. J Neurosurg 56:411-416, 1982

24. Singh D, Saxena A, Jagetia A, Singh H, Tandon MS, Ganjoo P: Endoscopic observations of blocked ventriculoperitoneal (VP) shunt: a step toward better understanding of shunt obstruction and its removal. Br J Neurosurg 26:747-753, 2012

25. Sotelo J: The hydrokinetic parameters of shunts for hydrocephalus might be inadequate. Surg Neurol Int 3:40, 2012

26. Stone JJ, Walker CT, Jacobson M, Phillips V, Silberstein HJ: Revision rate of pediatric ventriculoperitoneal shunts after 15 years. J Neurosurg Pediatr 11:15-19, 2013

27. Thomale UW, Hosch H, Koch A, Schulz M, Stoltenburg G, Haberl EJ, et al: Perforation holes in ventricular catheters-is less more? Childs Nerv Syst 26:781-789, 2010

\section{Disclosures}

This article is based on work performed using computational resources supported by the University of Tennessee and Oak Ridge National Laboratory Joint Institute for Computational Sciences (http://www.jics.tennessee.edu). Research described in this article was supported by the Science Alliance at the University of Tennessee, Knoxville, thorough the Joint Directed Research and Development (JDRD) Collaborative Cohort Program. Sofy Weisenberg and Stephanie TerMaath received financial and inkind support in the form of partial graduate student tuition and stipend, partial faculty summer salary, and computational hours on the supercomputer Darter.

This research used resources of the Oak Ridge Leadership Computing Facility at the Oak Ridge National Laboratory, which is supported by the Office of Science of the U.S. Department of Energy under Contract No. DE-AC05-00OR22725. The article was authored by UT-Battelle, LLC under the same contract with the U.S. Department of Energy. The United States Government retains and the publisher, by accepting the article for publication, acknowledges that the United States Government retains a nonexclusive, paid-up, irrevocable, worldwide license to publish or reproduce the published form of this manuscript, or allow others to do so, for United States Government purposes. The Department of Energy will provide public access to these results of federally sponsored research in accordance with the DOE Public Access Plan (http://energy.gov/downloads/doe-public-access-plan).

\section{Author Contributions}

Conception and design: Weisenberg, TerMaath, Barbier. Acquisition of data: Weisenberg, Barbier. Analysis and interpretation of data: Weisenberg, Barbier. Drafting the article: Weisenberg, TerMaath. Critically revising the article: Weisenberg, TerMaath, Barbier, Killeffer. Reviewed submitted version of manuscript: all authors. Approved the final version of the manuscript on behalf of all authors: Weisenberg. Statistical analysis: Weisenberg. Administrative/technical/material support: TerMaath, Barbier, Hill.

\section{Correspondence}

Sofy H. Weisenberg, Dougherty Engineering Bldg., 1512 Middle Dr., Rm. 523, Knoxville, TN 37996-2210. email: sweisenb@vols. utk.edu. 